\title{
ANOTHER PROOF OF SPIRA'S INEQUALITY AND ITS APPLICATION TO THE RIEMANN HYPOTHESIS
}

\author{
SADEGH NAZARDONYAVI AND SEMYON YAKUbOVICH
}

Abstract. By using new inequalities involving powers of rational functions, we give another proof of an important Spira's relation for the Riemann zeta-function $|\zeta(1-s)| \leqslant|\zeta(s)|$ in the strip $0<\Re s<1 / 2,|\mathfrak{I} s| \geqslant 12$. Moreover, we establish a sufficient condition of the validity of the Riemann hypothesis in terms of the derivative of $|\zeta(s)|^{2}$ with respect to $\Re s$ and conjecture its necessity.

Mathematics subject classification (2010): 11M26, 11M99, 26Dxx, 41A17, 33B15.

Keywords and phrases: Riemann zeta-function, size of the Riemann zeta-function, critical strip.

\section{REFERENCES}

[1] L. V. Ahlfors, Complex Analysis, An Introduction to the Theory of Analytic Functions of One Complex Variable, McGraw-Hill, Inc. 3rd edition, 1979.

[2] R. E. AtTAR, Special Functions and Orthogonal Polynomials, Lulu Press, 2006.

[3] R. D. Dixon AND LOWELl SCHOENFELD, The size of the Riemann zeta-function at places symmetric with respect to the Point 1/2, Duke Math. J. 33 (1966), 291-292.

[4] E. DUEÑEZ et. al., Roots of the derivative of the Riemann zeta function and of characteristic polynomials, Nonlinearity 23 (2010), 2599-2621.

[5] A. Ivić, The Riemann Zeta-Function, Dover Publications Inc., 2003.

[6] J. C. LAGARIAS, An elementary problem equivalent to the Riemann hypothesis, Amer. Math. Monthly 109, 6 (2002), 534-543.

[7] D. S. Mitrinović et. al., Elementary Inequalities, P. Noordhoff Ltd., Groningen, 1964.

[8] A. SPEISER, Geometrisches zur Riemannschen Zetafunktion, Math. Ann. 110 (1934), 514-521 (German).

[9] R. SPIRA, An inequality for the Riemann zeta function, Duke Math. J. 32 (1965), 247-250.

[10] E. C. Titchmarsh, The Theory of the Riemann Zeta-Function, 2nd edition, Clarendon Press Oxford University Press, Oxford, 1986. 